\title{
LA CAPACIDAD DE CORRECCIÓN DE ERRORES Y EL FEEDBACK CORRECTIVO ESCRITO: ESTUDIO DE CASOS DE APRENDICES DE PORTUGUÉS HISPANOHABLANTES
}

\author{
ERROR CORRECTION CAPACITY AND WRITTEN \\ CORRECTIVE FEEDBACK: CASES STUDY OF SPANISH \\ SPEAKERS LEARNERS OF PORTUGUESE
}

\section{Maria Rocio Alonso Rey ${ }^{*}$}

\section{RESUMEN}

La efectividad del feedback correctivo (FC) en la expresión escrita ha sido uno de los temas más controvertidos en las última décadas (BITCHENER y FERRIS, 2012; TRUSCOTT, 2007; VAN BEUNINGEN, 2010). Tras el predominio de los estudios experimentales de tipo cuantitativo preocupados por cuestiones de adquisición a largo plazo y centrados en una gama de errores muy restringida, se plantea la necesidad de prestar más atención a la mejora de la corrección de los textos o accuracy y a las posibilidades del feedback indirecto (FERRIS, 2010; STORCH, 2010). En este trabajo, se presenta un estudio de casos sobre la capacidad de detección de errores y los efectos del FC en la revisión de textos en hispanohablantes aprendices de portugués con diferentes grados de dominio de la lengua portuguesa. La capacidad de corrección de los errores, interpretada como proceso de detección de errores (noticing) y de recuperación de formas, es analizada mediante un test sin FC, un test con FC y un postest inmediato. Los resultados muestran que la capacidad de corrección mejora a muy corto plazo con la provisión de FC, si bien un conjunto de errores recuperables resultan no detectables para los aprendices.

Palabras clave: portugués lengua extranjera; feedback correctivo escrito; detección de errores.

\section{ABSTRACT}

The efectiveness of corrective feedback (CF) in written production has been controversial in the last decades (BITCHENER y FERRIS, 2012; TRUSCOTT, 2007; VAN BEUNINGEN, 2010). The investigation focused on experimental studies, with quantitative research, about long-term acquisition and with a restricted range of errors, but recently researchers make a call on the study of accuracy and indirect feedback (FERRIS, 2010; STORCH, 2010). This paper presents a cases study of error detection capacity and the corrective feedback effects on text editing in Spanish speakers learners of Portuguese with different levels of proficiency. Error correction, as the capacity of error detection and form recovery, is

\footnotetext{
* Universidad de Salamanca, Salamanca, Espanha. rocioalonsorey@usal.es 
analysed in a test without CF, a test with CF and a very short-term postest. The results show that error correction capacity improves but has no effect on a set of errors that the learners can recover but they can't notice.

Keywords: portuguese foreign language; written corrective feedback; noticing.

\section{INTRODUCCIÓN}

El efecto de la corrección en la expresión escrita es uno de los temas que más atención ha recibido en las últimas décadas y también uno de los más controvertidos (BITCHENER y FERRIS, 2012; FERRIS, 2004, 2010; POLIO, 2012; STORCH, 2010 TRUSCOTT, 2007; VAN BEUNINGEN, 2010). Es una práctica habitual en las clases de lengua extranjera que los profesores proporcionen feedback correctivo (FC) a los aprendices para que sean conscientes de las formas incorrectas utilizadas y las puedan producir adecuadamente en lo sucesivo. Sin embargo, la efectividad de esta práctica de provisión de feedback ha sido cuestionada desde los 90, en que se planteaba el denominado "caso contra la corrección" o "debate Truscott" (BRUNTON, 2009), que contempla la posibilidad de que la corrección sea inefectiva, innecesaria o incluso pueda llegar a ser contraproducente (TRUSCOTT, 1996, 1999 2004, 2007, 2009, 2010; TRUSCOTT y HSU, 2008). Estas afirmaciones han generado un intenso debate ${ }^{1}$ entre detractores y defensores del uso del FC en la expresión escrita, estos últimos planteando, por oposición, el "caso a favor de la corrección" (FERRIS, 2002).

Entre los defensores del FC se hallan dos perspectivas diferentes, la primera de ellas corresponde a los estudios tempranos denominados como feedback for edition (VAN BEUNINGEN, 2010; WILLIAMS, 2012) o investigación en L2 writting (FERRIS, 2010). Este tipo de estudios considera el FC como una herramienta para mejorar la corrección de la escritura en el conjunto del texto y sigue una metodología de investigación que se desarrolla en tres estadios: redacción del texto - aplicación del FC - revisión del texto. Partiendo de una primera versión del texto escrito, tras la provisión del FC, se miden los efectos del FC en la corrección del texto inicial, esto es, la respuesta inmediata al FC.

Estos estudios tempranos recibieron fuertes críticas (ELLIS et al. 2008; FERRIS, 2010; TRUSCOTT, 2007) en dos aspectos principales: por un lado, en lo que se refiere a su diseño poco controlado e inconsistente en ocasiones; por otro

\footnotetext{
1 Véase por ejemplo cómo la posición planteada en Truscott (1996) es directamente contestada por Ferris (1999), Chandler $(2003,2009)$ o Brunton (2009) a quienes a su vez responde el primero en Truscott $(1999,2004$ y 2009) respectivamente.
} 
lado, por el hecho de no demostrar que esas correcciones se retengan a largo plazo y se apliquen a nuevos textos.

La segunda perspectiva a favor del FC, denominada feedback for learning, se centra en la aportación del FC a la adquisición y, en la pasada década, conseguía probar, en condiciones experimentales, la efectividad del FC en el aprendizaje de L2 a largo plazo (BITCHENER et al., 2005; BITCHENER y KNOCH 2008, 2009a, 2009b; SHEEN, 2007). La metodología de investigación incorpora un pretest con la producción de un texto, un postest tras la provisión de FC y un delayed postest, poniendo a prueba los efectos del FC en la producción de nuevos textos escritos (ELLIS et al., 2008). Sin embargo, los resultados obtenidos solo confirman esa efectividad del feedback de modo parcial y en circunstancias muy específicas. Para Truscott (2010) a pesar de que se ha estudiado en un amplio número de casos, la corrección parece tener unos efectos positivos demasiado pequeños $y$, de hecho, la efectividad del FC se encuentra en circunstancias muy restringidas: (i) asociada a un tipo de FC directo y explícito, de preferencia complementado con entrevistas para clarificar y explicar los errores o con explicaciones escritas (BITCHENER y KNOCH, 2008; SHEEN, 2007) y (ii) aplicado a unos pocos elementos, uno o dos rasgos lingüísticos, sobre los que se focaliza la acción correctiva, tales como el artículo o el pasado simple (v. STORCH, 2010, para una revisión de estudios). Asimismo, estas propuestas del feedback for learning parecen alejadas y poco realistas a la hora de llevarlas a la práctica en situaciones reales de enseñanza en el aula (FERRIS, 2010). En lo que respecta al FC directo no explícito no está claro que los beneficios de la corrección se extiendan a nuevas tareas de escritura (TRUSCOTT y HSU, 2008).

Tales resultados justifican la necesidad de revisar los condicionantes que restringen la efectividad del FC. En los orígenes del debate, Truscott (1996) apuntaba como motivos principales de esta inefectividad la naturaleza del proceso de corrección y la propia complejidad del proceso de aprendizaje. Este trabajo se centra en el primero y recoge los planteamientos del feedback for edition para observar y analizar las limitaciones que surgen cuando el aprendiz se enfrenta al error. En concreto en la capacidad de identificar o apercibirse del error y en la naturaleza de comprender la naturaleza del error.

\section{ESTUDIOS RECIENTES SOBRE RECONOCIMIENTO Y COMPRENSIÓN DEL FC ESCRITO}

En la presente década, tras el predominio de los estudios experimentales de tipo cuantitativo preocupados por cuestiones de adquisición a largo plazo 
y centrados en una gama de errores muy restringida, se plantea la necesidad de prestar más atención a la mejora de la corrección de los textos o accuracy y a las posibilidades del feedback indirecto (STORCH, 2010; FERRIS, 2010).

Hay consenso en que la corrección ayuda a los estudiantes a reducir sus errores sustancialmente en el texto en que reciben esas correcciones, aceptado también por los detractores de la corrección (v. TRUSCOTT y HSU, 2008). Se considera que la investigación del proceso de corrección puede proporcionar informaciones útiles para el estudio de los efectos del FC (FERRIS y ROBERTS, 2001) y se asume la existencia de dos aspectos fundamentales en este proceso: una primera dimensión que tiene que ver con la capacidad de identificar el error y una segunda dimensión que tiene que ver con la capacidad de comprender la naturaleza de ese error. Tal y como señalan Mackey et al. (2000, p. 477) "it is important to first investigate the extent to which that feedback is in fact perceived as such by learners and whether their perceptions about the target of the feedback are correct".

La capacidad de identificar el error tiene que ver con la posibilidad que el aprendiz tiene de apercibirse o "notar" que una forma que ha utilizado es incorrecta. Esta primera dimensión es explorada por una de las perspectivas teóricas en que se ha basado el estudio del FC: la consideración de este como noticing facilitator (VAN BEUNINGEN, 2010), esto es, como forma que facilita que el aprendiz perciba su uso de formas desviadas. Esta perspectiva se basa en la "Hipótesis del noticing" de Schmidt $(1990,2001)$ que establece que una condición para el aprendizaje es el noticing, el notar, reparar o apercibirse, de forma consciente, de que el output no corresponde con lo esperable en la lengua objeto (LO). Este fenómeno se relaciona además con dos aspectos concretos: la atención (attention), entendida como la capacidad de que los aprendices adviertan la no correspondencia y la alerta (awareness), cuando los aprendices son conscientes de los rasgos que marcan esa diferencia, que se apoyaría en un tipo de conocimiento metalingüístico explícito.

En esta línea, Qi y Lapkin (2001) conducen un estudio de casos $(\mathrm{n}=2)$, desarrollado en los tres estadios de la metodología del feedback for editing, en el que utilizan un tipo de FC indirecto que consiste en la reformulación del texto. Siguiendo el protocolo de pensar en voz alta (think-aloud protocol), analizan los comentarios de los aprendices para investigar la profundidad del procesamiento de este tipo de FC. Concluyen que el FC mejora la capacidad de detección de los errores o noticing y apuntan que el tipo o calidad del noticing puede ser un factor determinante. El reconocimiento no comprensivo (esto es, sin razones o explicaciones claramente articuladas) tiene un menor impacto en la retención: el aprendiz con un nivel más bajo, que muestra este tipo de detección, tiene más dificultades en retener el FC, 
mientras que el aprendiz de nivel más alto muestra un tipo de noticing comprensivo y un mayor grado de aprovechamiento del FC, resultados que los autores relacionan también con unos conocimientos lingüísticos más amplios. Encuentran además que, aun habiendo una mejora sustancial de la corrección en ambos casos, no todas las

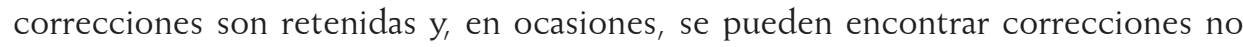
recogidas en el FC.

En relación con la comprensión del error, Han (2001) en un estudio de caso $(n=1)$ encuentra que la interpretación del error por parte del profesor puede no coincidir con el problema de aprendizaje del estudiante; en consecuencia, el aprendiz falla en el reconocimiento de la diferencia entre su output y la corrección del profesor. Así se establece como condición para la efectividad del FC una sintonía fina (fine tumning) entre ambos, esto es, debe haber una congruencia entre la intención del hablante y la interpretación del oyente, así como entre el contenido de la corrección y la receptividad del aprendiz para procesarlo.

Sach y Polio (2007) en un estudio también de tres estadios con protocolo de pensamiento en voz alta, comparan dos tipos de FC, reformulación y corrección directa y explícita de errores. Los resultados indican que este último tipo de corrección de errores conlleva una mejora en la revisión superior a la obtenida mediante la reformulación. En lo que respecta a la calidad o tipo de noticing confirman los resultados de Qi y Lapkin (2001): el noticing con comprensión o "sustantivo" es retenido en mayor medida que el noticing sin comprensión o "perfunctorio". Los autores relacionan igualmente estos resultados con los conocimientos lingüísticos del aprendiz, pero también con su capacidad para verbalizar y hablar sobre ellos. Sin embargo, no todas las correcciones sustantivas son incorporadas y a veces los aprendices incorporan correcciones no indicadas.

Storch y Wigglesworth (2010), por su parte, analizan los resultados del procesamiento, uptake y retención del FC directo (codificado) e indirecto (reformulación) en un estudio de casos, con la intención de establecer la relación entre la retención y la comprensión. Esta relación se formula en términos de engagement, esto es, implicación o compromiso, de tipo extensivo, cuando se ofrecen explicaciones o hay algún tipo de referencia de conocimiento metalingüístico, o limitado, cuando solo se lee o repite el FC. Según sus resultados, el FC directo conduce a más modificaciones que la reformulación y el grado de compromiso también es mayor con este tipo de feedback. Asimismo, la comprensión y la implicación llevan a más casos de retención, si bien esta parece también depender de la naturaleza del error. Por ejemplo, en los errores superficiales (ortografía), el noticing no comprensivo, verbalizado o no, puede ser suficiente para la retención. Su 
conclusión es que la efectividad del FC está relacionada con la interacción, dinámica y compleja, de aspectos tanto lingüísticos como afectivos.

En el ámbito del Portugués para Hablantes Español (PHE) muy pocos estudios han centrado su atención en esta materia, a pesar del reconocimiento de la importancia del FC (ALMEIDA, 2004; GRANNIER, 2012). Con todo, en relación con el uso del conocimiento metalingüístico, cabe reseñar el estudio de Carvalho y Silva (2008) en el que, utilizando el protocolo de pensamiento en voz alta, encuentran que los aprendices hispanohablantes descansan menos en su conocimiento metalingüístico que los aprendices con otras LM en la realización de actividades gramaticales.

\section{DISEÑO DE INVESTIGACIÓN}

\subsection{Objetivos del análisis}

El objetivo aquí es explorar los efectos del FC en la capacidad de corrección de un texto, específicamente en dos de los aspectos considerados relevantes en este proceso: la capacidad de identificar el error y la capacidad de reconocer o comprender el desvío.

Las preguntas que se plantean son:

- cómo influye la provisión de FC implícito en la capacidad de corrección de los errores de un texto y, en particular, en la capacidad de detección de esos errores.

- qué tipo de reconocimiento de la naturaleza de sus errores realizan.

\subsection{Participantes}

Forman parte de esta investigación 7 estudiantes del Grado en Estudios Portugueses y Brasileños/Licenciatura en Filología Portuguesa de la Universidad de Salamanca (USAL) que completaron 4 semestres de formación obligatoria en Lengua Portuguesa y que cursaban el $5^{\circ}$ semestre en el momento de realización del estudio. En lo que respecta a sus conocimientos lingüísticos, los casos 1, 2, 3, 4,5 y 6 tienen el español como lengua materna (E/LM) mientras que el caso 7 es hablante nativo de alemán y domina el español como L2. Por otro lado, 2 de los participantes con español como LM tienen un nivel alto de portugués: en el caso 1 al ser lengua de herencia (LH) mientras que el el caso 2 es un bilingüe tardío (con 7 años de residencia en Portugal de los 14 a los 21 años). Ambos se encuadrarían 
globalmente en el nivel C2 del Marco Europeo de Referencia (2003), si bien el caso 1 indica que su nivel en producción escrita es un B2 mientras que el caso 2 indica un nivel $\mathrm{C}_{2}^{2}$. De los restantes aprendices E/LM, encuadrados en el nivel B1/2: los casos 3 y 4 (indicados como L2) han tenido experiencias lingüísticas de inmersión (becas de estudio en países de habla portuguesa) a diferencia de los casos 5 y 6 (indicados como LE).

Cuadro 1. Caracterización lingüística de los participantes

\begin{tabular}{|c|c|c|c|c|}
\hline Informante & Español & Portugués & $\begin{array}{c}\text { Autoevaluación } \\
\text { producción escrita }\end{array}$ & Nivel de lengua \\
\hline Caso 1 & LM & Bilingüe (LH) & B2 & C2 \\
\hline Caso 2 & LM & Bilingüe (tardío) & C2 & C2 \\
\hline Caso 3 & LM & L2 & B2 & B2 \\
\hline Caso 4 & LM & L2 & B1 & B1 \\
\hline Caso 5 & LM & LE & A2 & B1 \\
\hline Caso 6 & LM & LE & B1 & B1 \\
\hline Caso 7 & L2 & L2 & C1 & B2 \\
\hline
\end{tabular}

\subsection{Metodología, procedimiento y análisis de los datos}

Dado que el objetivo aquí no es probar los efectos del FC en el aprendizaje sino las limitaciones que este presenta en su retención a corto plazo y su relación con la comprensión del error, para explorar la capacidad de detección y la percepción de la naturaleza del error se sigue aquí el diseño de los estudios clásicos de revisión de textos (FERRIS, 2010, p. 195): redacción de un texto - provisión de FC - revisión del texto. En este diseño original se introducen un test complementario (Cuadro 2).

\section{Cuadro 2. Diseño de investigación}

\begin{tabular}{|c|c|c|}
\hline & Diseño Clasico & Diseño del estudio \\
\hline Redacción & Pretest & Prestest \\
\hline Provisión de FC & Test con FC & Test sin FC (test 1) \\
\cline { 2 - 3 } & Postest & Test con FC (test 2) \\
\hline Revisión & Postest (test 3) \\
\hline
\end{tabular}

El test 1 permite determinar la capacidad de corrección que los aprendices poseen sin FC, aspecto que no se contempla en este tipo de estudios, donde se da por sentado que los aprendices lo han hecho "lo mejor posible" en la producción

2 Los informantes dispusieron de la hoja de autoevaluación que forma parte del Portafolio para determinar su nivel en las 4 destrezas. 
de ese texto (v. QUI y LAPKIN, 2001, p. 285) y, por tanto, se asume que no reconocerán sus propios errores sin el auxilio del FC. Sin embargo, estudios en el ámbito del PHE indican que es posible que los aprendices no estén haciendo uso de sus conocimientos metalingüísticos a la hora de realizar actividades de producción controladas (CARVALHO y SILVA, 2008). La hipótesis que aquí se baraja es que no prestan atención (en los términos de la hipótesis del noticing) y no usan esos conocimientos en la revisión de sus textos, si bien podrían hacerlo y, por tanto, corregir algunos de esos errores sin FC. Por otro lado, en el test 2, tras la provisión de FC implícito, se sustituye el protocolo de pensar en voz alta por una hoja de respuesta, en que se pregunta explícitamente por la explicación del error que conduce a la corrección. De esta forma, se obliga al aprendiz a reflexionar sobre el error, para obtener así datos sobre la percepción de la naturaleza del error. En el postest, se somete nuevamente a revisión el texto para comprobar la retención inmediata de ese FC.

La primera semana los participantes fueron informados de los detalles de la investigación y dieron su consentimiento para participar en ella. A continuación rellenaron una ficha para determinar su perfil lingüístico. Se utilizó para tal fin el "Pasaporte de lenguas" del Portafolio Europeo de Lenguas, y realizaron el pretest, que consistía en la redacción de un texto sobre su día a día durante el curso académico.

El texto original fue recogido y reproducido en un documento word que se entregó a dos correctores (profesores nativos de lengua portuguesa de la USAL) para su revisión, con la indicación de que señalasen los errores encontrados. El grado de coincidencia fue de $73 \%$, por lo que fue entregado a un tercer corrector, la investigadora, que analizó los casos de discordancia y resolvió las discrepancias. Las complicaciones surgidas en este punto obligaron a retrasar en una semana el calendario previsto para los siguientes test. La tercera semana, los participantes realizaron un test de corrección (test 1): recibieron una copia de su texto (pretest) para indicar y corregir sus errores. La cuarta semana, recibieron el FC y realizaron la corrección del texto (test 2): se les entregó una copia del texto con FC indirecto sin codificar, con los errores destacados en negrita y subrayados, y una hoja de respuesta con la lista de formas desviadas, donde debían apuntar la corrección y comentar el error, indicando qué estaba mal y por qué. Una vez recogido el test 2, se les entregó el postest: una copia del texto original (sin FC) para que indicasen las correcciones que harían en la versión definitiva de ese texto sobre su día a día ${ }^{3}$.

3 Una vez terminada y recogida la prueba, se comentaron en el aula los desvíos no identificados por los aprendices mostrando y explicando las formas/reglas correctas a partir del documento con FC. 
Para el análisis de los datos, a partir del pretest se crea una lista de desvíos o formas de superficie erróneas en las cuales se analiza y localiza el error o errores ${ }^{4}$ y se introducen en una tabla Excel. A continuación se introducen los resultados del tratamiento dado a esos errores en los test, clasificados en tres grandes clases: correcciones adecuadas (CA), formas no corregidas (NC) y formas corregidas inadecuadamente $(\mathrm{CI})$. La capacidad de corrección para cada uno de los test se establece a partir del valor obtenido al calcular la frecuencia de CA en base al número de errores encontrados en cada aprendiz.

\section{RESULTADOS DEL ANÁLISIS}

\subsection{Pretest}

En el análisis de los textos (Anexo I) se encontraron, en las 897 palabras utilizadas, un total de 106 formas desviadas que corresponden a 112 errores (Tabla 1).

Tabla 1. Resultados del pretest

\begin{tabular}{|c|c|c|c|c|}
\hline & Desvíos & Errores & N $^{\text {o Palabras }}$ & Frec E \\
\hline Caso 1 & 10 & 10 & 160 & 0.06 \\
\hline Caso 2 & 7 & 7 & 105 & 0.07 \\
\hline Caso 3 & 17 & 17 & 193 & 0.09 \\
\hline Caso 4 & 21 & 22 & 133 & 0.17 \\
\hline Caso 5 & 13 & 16 & 60 & 0.27 \\
\hline Caso 6 & 25 & 27 & 126 & 0.21 \\
\hline Caso 7 & 13 & 13 & 120 & 0.11 \\
\hline
\end{tabular}

La distribución de errores y su frecuencia (Frec E) varía según los casos, con un menor número de errores en los aprendices con un nivel más alto y con mayor exposición a la L2.

\subsection{Test 1: corrección sin feedback}

En el test de corrección sin feedback, con un total de 112 errores, se encuentran 14 correcciones adecuadas (CA) y 95 casos sin corrección (NC). Se incluyen aquí

4 Hay desvíos (palabras que no corresponden con la forma o uso de la LO) que combinan varios errores, por ejemplo *es (utilizado en lugar de é) es un desvío que presenta un error gramatical y un error ortográfico en la acentuación. 
algunos errores que son detectados por el informante pero este no es capaz de ofrecer la forma correcta en la LO $(n=4)$. En la Tabla 2 se presentan los resultados con indicación del valor de la frecuencia de corrección con el que se establece la medida de la capacidad de corrección (CapC).

Tabla 2. Resultados del test 1

\begin{tabular}{|c|c|c|c|c|}
\hline & CA & NC & CI & CapC \\
\hline Caso 1 & 3 & 7 & 0 & 0.43 \\
\hline Caso 2 & 1 & 6 & 0 & 0.14 \\
\hline Caso 3 & 0 & 17 & 0 & 0.00 \\
\hline Caso 4 & 2 & 20 & 0 & 0.09 \\
\hline Caso 5 & 7 & 9 & 0 & 0.44 \\
\hline Caso 6 & 0 & 25 & 2 & 0.00 \\
\hline Caso 7 & 2 & 11 & 0 & 0.15 \\
\hline
\end{tabular}

Además de los resultados indicados, se encuentran también correcciones no requeridas (NR), que no inciden sobre aspectos incorrectos. La introducción de estas correcciones tiene un efecto neutro en la mayoría de los casos $(n=4)$, esto es, no afecta a la corrección (por ejemplo, sustitución de "as tarefas" por "os TPC's"). Sin embargo, en uno de los casos, supone la introducción de una forma incorrecta, concretamente el informante 2 coloca la forma *vesto (para visto) seguida de una interrogación.

\subsection{Test 2: corrección con feedback directo implícito no codificado}

En el segundo test, con provisión de feedback directo implícito no codificado (Tabla 3), del total de 112 errores fueron corregidos adecuadamente 70. Se encuentran, además, correcciones que llevan aparejados nuevos errores: casos $\mathrm{C}+$ $(\mathrm{n}=3)$. A pesar de la provisión de FC implícito, en ocasiones los informantes no son capaces de indicar la forma correcta $(n=19)$ o la corrección es inadecuada $(n=19)$, junto con un caso en que un participante opta por la reformulación (Ref) con supresión del elemento problemático, por lo que se contabiliza con NC. 
Tabla 3. Resultados del test 2

\begin{tabular}{|c|c|c|c|c|c|c|}
\hline & \multicolumn{2}{|c|}{ CA } & \multicolumn{2}{c|}{ NC } & \multirow{2}{*}{ CI } & \multirow{2}{*}{ CapC } \\
\cline { 2 - 5 } & CA & C+ & NC & Ref & & \\
\hline Caso 1 & 8 & 0 & 2 & 0 & 0 & 0.43 \\
\hline Caso 2 & 6 & 0 & 1 & 0 & 0 & 0.14 \\
\hline Caso 3 & 5 & 0 & 0 & 0 & 12 & 0.00 \\
\hline Caso 4 & 11 & 0 & 9 & 0 & 2 & 0.09 \\
\hline Caso 5 & 14 & 0 & 1 & 0 & 1 & 0.44 \\
\hline Caso 6 & 17 & 3 & 6 & 0 & 1 & 0.00 \\
\hline Caso 7 & 9 & 0 & 0 & 1 & 3 & 0.15 \\
\hline
\end{tabular}

En el apartado de comentario del test, donde se pide a los aprendices que indiquen qué estaba mal y los motivos, tales como la regla a aplicar, se obtienen un total de 99 comentarios. En el 83\% de los casos el comentario consiste en una descripción del cambio realizado, sin motivar. Apenas en el 17\% de los comentarios se explica la regla aplicada o se describe la causa del error (Tabla 4).

Tabla 4. Tipo de comentarios en el test 2

\begin{tabular}{|c|c|c|}
\hline & Descripción & Motivación \\
\hline Caso 1 & 7 & 3 \\
\hline Caso 2 & 6 & 0 \\
\hline Caso 3 & 16 & 0 \\
\hline Caso 4 & 8 & 0 \\
\hline Caso 5 & 13 & 2 \\
\hline Caso 6 & 20 & 10 \\
\hline Caso 7 & 12 & 2 \\
\hline
\end{tabular}

En los casos 2, 3 y 4 solo se describe formalmente el cambio realizado, mientras que 1,5,6 y 7 proporcionan algún tipo de explicación. Los comentarios (Anexo II) son, a grandes rasgos, bastante generales e imprecisos en su conjunto, del tipo "acentuación", "ortografía", "se escribe con [...]". Además, en las descripciones se encuentran algunos errores de interpretación, tales como confusión de las nociones de tiempo y persona (6.11) o confusión entre cuestiones ortográficas y gramaticales (3.18). Cabe también destacar la formulación de reglas ad boc (6.2 y 6.14.), en que el aprendiz formula una regla basada en su observación del fenómeno que no se corresponde con la regla de la lengua objeto: "As horas obrigam acentuar a preposição" en el caso de la contracción de preposición y artículo. 


\subsection{Postest}

En el test 3 (Tabla 5) hay 61 correcciones adecuadas, 32 errores sin corregir y 19 casos de correcciones inadecuadas.

Tabla 5. Resultados del postest

\begin{tabular}{|c|c|c|c|c|c|c|}
\hline & \multicolumn{2}{|c|}{ CA } & \multicolumn{2}{c|}{ NC } & \multirow{2}{*}{ CI } & \multirow{2}{*}{ CapC } \\
\cline { 2 - 6 } & CA & C + & NC & Ref & & \\
\hline Caso 1 & 8 & 0 & 2 & 0 & 0 & 0.43 \\
\hline Caso 2 & 6 & 0 & 1 & 0 & 0 & 0.14 \\
\hline Caso 3 & 4 & 0 & 2 & 0 & 12 & 0.00 \\
\hline Caso 4 & 12 & 0 & 6 & 2 & 2 & 0.09 \\
\hline Caso 5 & 8 & 0 & 2 & 4 & 1 & 0.44 \\
\hline Caso 6 & 13 & 4 & 9 & 0 & 1 & 0.00 \\
\hline Caso 7 & 6 & 0 & 3 & 1 & 3 & 0.15 \\
\hline & 57 & 4 & 25 & 7 & \multirow{2}{*}{32} & \\
\cline { 1 - 4 } & 61 & & 32 & \\
\hline
\end{tabular}

Dentro de las correcciones adecuadas (CA), se encuentran 4 correcciones, indicadas como $\mathrm{C}+$, que traen aparejadas incorrecciones en otro nivel de análisis lingüístico, del tipo *torno (error léxico) $>*^{*}$ regreso (error ortográfico). Por otro lado, en el grupo de resultados sin corrección del error, se encuentran casos en que el informante opta por reformular el enunciado (Ref) suprimiendo o cambiando parte de sus elementos con lo que se modifica sustancialmente su contenido.

\section{DISCUSIÓN}

\subsection{Test 1}

Las formas detectadas y corregidas muestran que algunos aprendices (casos $1,2,4,5$ y 7 ) confrontados con su texto y sin recibir FC, son capaces de corregir algunos de sus errores. Sin embargo, esa capacidad de autocorrección no está presente en todos los sujetos (casos 3 y 6 ) y no parece estar relacionada con el nivel lingüístico ni el tipo de exposición. Esto indica que los primeros no han explotado su capacidad de autocorrección del texto en el pretest, como fase de edición que forma parte de la tarea de elaboración de un texto, si bien tendrían la capacidad para detectar y corregir algunos de esos errores. 
La falta de aprovechamiento de esta capacidad puede ser debida a:

(i) aspectos relacionados con el proceso de edición (como fase en la elaboración del texto) que puede obedecer a (i.a) una revisión superficial, por falta de compromiso con la calidad de la producción, (i.b) una revisión mal focalizada, por el uso de estrategias inadecuadas de revisión;

(ii) aspectos relacionados con la capacidad de corrección, tal como un bajo nivel de alerta ante la aparición de desvíos, que en el caso de hispanohablantes se relaciona con el mito de la facilidad (ALMEIDA, 1995; ALONSO REY, 2005, 2012; FERREIRA, 1995), esto es, la creencia de los aprendices de que el portugués es una lengua que requiere poco esfuerzo para el hablantes de español, que consigue aprender y comunicarse en esta lengua naturalmente, de forma rápida y efectiva.

\subsection{Test 2}

La provisión de FC implícito supone una mejora en la corrección del texto si lo comparamos con la tarea realizada sin FC (Tabla 6).

Tabla 6. Comparativa de la capacidad de corrección en los test 1 y 2

\begin{tabular}{|c|c|c|}
\hline & Test 1 & Test 2 \\
\hline Caso 1 & 0.4 & 0.8 \\
\hline Caso 2 & 0.1 & 0.9 \\
\hline Caso 3 & 0.0 & 0.3 \\
\hline Caso 4 & 0.1 & 0.5 \\
\hline Caso 5 & 0.4 & 0.9 \\
\hline Caso 6 & 0.0 & 0.6 \\
\hline Caso 7 & 0.2 & 0.7 \\
\hline
\end{tabular}

Los errores $\mathrm{NC}$ y $\mathrm{CI}$ suponen la existencia de errores que los aprendices

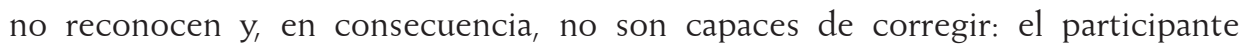
no comprende el error y no es capaz de recuperar la forma correcta. En el caso específico de $\mathrm{CI}$, se puede observar el fenónemo de falta de tunning (HAN, 2001) o congruencia entre el FC implícito y la interpretación del aprendiz.

En lo que se refiere a los casos corregidos adecuadamente (CA) cabría suponer que los aprendices han comprendido el FC. Sin embargo, a partir de los comentarios incluidos en la hoja de respuestas, se observa que las explicaciones 
que ofrecen los participantes consisten mayoritariamente en señalar el tipo de error (por ejemplo, acentuación) o describir la alteración (sin o, no es necesaria la preposición,...). En pocos casos se indica el motivo (interferencia, está en español) o la regla a aplicar (as palavras em-agem são femininas).

En las primeras no está claro que se trate necesariamente de correcciones comprensivas, esto es, basadas en reglas/conocimientos explícitos de L2. Pueden ser realizadas en base a intuiciones lingüísticas y ser categorizadas a posteriori. El caso 5 es bastante ilustrativo: para *bici se propone bicicleta, forma correcta en L2, pero el participante indica que, en realidad, "no sé cómo es en portugués". En una conversación informal con los aprendices, estos reconocieron a la investigadora que, por ejemplo, no son capaces de formular las reglas de acentuación.

Llama la atención la imprecisión, falta de rigor en el uso de la terminología metalingüística y la descuidada expresión en la descripción y la formulación de reglas (impropia en algunos casos por su simplicidad de estudiantes de filología). Este fenómeno se puede relacionar, por un lado, con el grado de compromiso en la realización y explicación de las correcciones pero también, por otro lado, con un desarrollo bajo de la capacidad de reflexión metalingüística y el desconocimiento o conocimiento vago de la terminología correspondiente, en este sentido apuntarían las incorrecciones en el uso de algunos términos.

\subsection{Postest}

En el postest, sin el apoyo del FC, la capacidad de corrección es menor pero mejora en relación a los resultados del test 1 .

Tabla 7. Comparativa de la capacidad de corrección en los tres tests

\begin{tabular}{|c|c|c|c|}
\hline & Test 1 & Test 2 & Test 3 \\
\hline Caso 1 & 0.4 & 0.8 & 0.8 \\
\hline Caso 2 & 0.1 & 0.9 & 0.9 \\
\hline Caso 3 & 0.0 & 0.3 & 0.2 \\
\hline Caso 4 & 0.1 & 0.5 & 0.5 \\
\hline Caso 5 & 0.4 & 0.9 & 0.5 \\
\hline Caso 6 & 0.0 & 0.6 & 0.5 \\
\hline Caso 7 & 0.2 & 0.7 & 0.5 \\
\hline
\end{tabular}

En el postest (Tabla 7), en términos generales, los errores CI y NC se mantienen como tales en comparación con los resultados del test 2 . No obstante, en 
el caso de los errores $\mathrm{NC}$ se encuentran resultados en que los aprendices han tomado conciencia de la existencia de formas incorrectas, aunque desconocen la forma adecuada. Esta huella del FC se materializa en reformulaciones con eliminación, en el caso $4 *$ ja $>0$ y $*$ durmo $>0$, o con sustitución, como ${ }^{*}$ gimnastica $>*$ desporte en el caso 5 .

Por su parte, los errores CA son también corregidos adecuadamente, en general, en el test 3, esto es, la forma correcta es retenida. Sin embargo, se encuentra un conjunto de errores recuperables que no son detectados, esto es, sobre los que el FC es inefectivo y pasan inadvertidos. Estos errores (Cuadro 3) no son retenidos por los aprendices a pesar de que poseen los conocimientos necesarios.

Cuadro 3. Errores inadvertidos en el test 3 con indicación de la forma en la interlengua (IL) y en la lengua objeto (LO)

\begin{tabular}{|c|c|c|}
\hline Casos & Forma de IL & Forma de la LO \\
\hline 3 & *ao dia seguinte & no dia seguinte \\
\hline \multirow{2}{*}{5} & $*_{\mathrm{O}}$ & ou \\
\hline & *alguma filme & algum filme \\
\hline \multirow{3}{*}{6} & *manha & manhã \\
\hline & $*_{\mathrm{O}}$ & ou \\
\hline & * gimnasio & ginásio \\
\hline \multirow{3}{*}{7} & *tengo & tenho \\
\hline & *cuando & quando \\
\hline & $*_{\mathrm{o}}$ & ou \\
\hline
\end{tabular}

Estas formas no retenidas corresponden principalmente a cuestiones ortográficas $(\mathrm{n}=5)$ tales como la forma gráfica de la conjunción disyuntiva y del grupo [kwa] o la representación formal de la nasalidad vocálica; cuestiones de selección de base léxica $(n=2)$ en el caso del error léxico * gimnasio y del error morfológico *tengo. Con la excepción de *alguma filme, con un error en el rasgo de género $(n=1), y$ *ao dia seguinte, con un error en la selección de preposición.

Los errores inadvertidos no se encuentran en los informantes con un nivel más alto (casos 1 y 2) y que tienen un menor número de errores. En estos casos es probable que tanto su competencia lingüística de la lengua como el número bajo de errores les permita un mayor dominio de su producción y la edición del texto. 


\section{CONCLUSIONES}

La corrección de errores puede ser considerada como una habilidad que implica dos procesos diferenciados (1) detección y (2) recuperación. El primero se refiere a la capacidad de detectar o reconocer el error como forma desviada de la LO, lo que se conoce como noticing: el aprendiz encuentra un elemento que percibe como no adecuado o correcto en la LO, una especie de percepción de que algo no encaja. Esta percepción puede ser inducida por el FC aunque no vaya asociada a conocimientos explícitos. Por otro lado, como segundo proceso, actúa la recuperación de la forma correcta. Esta puede estar basada (a) en la comprensión de una regla o de una diferencia entre L1 y L2, esto es, en conocimientos estables y objetivos de la L2, que pueden ser explicitados por el aprendiz - noticing comprensivoo bien (b) en impresiones o percepciones, de naturaleza subjetiva (tales como la regla de acentuación de las horas en el caso 6), o en conocimientos inestables (por ejemplo, en el caso 5 de la CA de *bici a bicicleta en la que el aprendiz reconoce que no sabe cómo es en portugués y que, en el test 3, opta por eliminar) - noticing no comprensivo-.

Los resultados indican, asimismo, que algunos aprendices no explotan su potencial de detección de errores en el proceso elaboración del texto, en su revisión inmediata, pero sí cuando se les insta a hacer esa revisión más profunda. Estos casos plantean la posibilidad de que sea necesario trabajar con estos aprendices la "alerta" ante los desvíos y su implicación o compromiso con la corrección del texto.

En lo que respecta a la recuperación o tipo de noticing, las correcciones de gran parte de esos errores no se asocian a noticing comprensivo, sino que parecen corresponder a impresiones subjetivas o conocimientos inestables más que a conocimientos objetivos y explícitos de la L2. Este tipo de recuperación no comprensiva es la que en ocasiones puede llevar a correcciones inadecuadas $\mathrm{o}$, puntualmente, a corregir elementos correctos. Por otro lado, aunque gran parte de las correcciones son retenidas a corto plazo, hay algunos elementos que presentan problemas de detección, son errores que continúan inadvertidos o invisibles, fundamentalmente de tipo ortográfico y relacionados con la forma léxica. Un fenómeno cuya explicación cabría profundizar en futuros estudios.

En conclusión, con la aplicación del FC implícito, los aprendices han sido capaces de corregir algunos errores que sin FC no habían sido capaces de detectar, en este sentido el FC es útil a muy corto plazo para mejorar la corrección del texto. El problema que se ha planteado en diferentes estudios es si esa mejora perdura a largo plazo y contribuye al aprendizaje y se ha centrado en tipos de errores que 
efectivamente mejoran con el FC. La posibilidad que aquí se plantea, a la luz de los datos obtenidos, es que el FC puede estimular la detección mientras que la recuperación dependerá del conocimiento estable de la lengua objeto. En otros términos, el FC activaría y ayudaría a desarrollar la alerta o sensibilidad hacia las discrepancias con la $\mathrm{LO}$ en el texto producido, mientras que la recuperación de las formas correctas (y su fijación) depende de la naturaleza y organización de los conocimientos de la $\mathrm{LO}$ que posee el aprendiz.

\section{REFERENCIAS BIBLIOGRÁFICAS}

ALMEIDA FILHO, J. C. (1995). Uma metodologia específica para o ensino de línguas próximas? En J. C. Almeida Filho, Português para estrangeiros interface com o espanbol. Campinas: Pontes, pp. 13-21.

ALMEIDA FILHO, J. C. (2004). Questões da interlíngua de aprendizes de português a partir ou com a interposição do espanhol (língua muito próxima). En A. R. Simões, A. M. Carvalho y L. Wiedemann, Portuquês para falantes de espanbol. Campinas: Pontes, pp. 183-191.

ALONSO REY, M.R. (2005). El mito de la facilidad en el estudio del proceso de enseñanza/ aprendizaje de PLE-HE. Estudios Portugueses, 4, pp. 11-38.

ALONSO REY, M.R. (2012). La transferencia en el aprendizaje de portugués por bispanobablantes. Salamanca: Luso-Española de Ediciones.

FERREIRA, I. (1995). A interlíngua do falante de espanhol e o papel do professor: aceitação tácita ou ajuda para superá-la? En J. C. Almeida Filho (org.), Português para estrangeiros interface com os espanbol. Campinas: Pontes, pp. 39-48

BITCHENER, J. y FERRIS, D. (2012). Written corrective feedback in second language acduisition and writing. Nueva York: Routledge.

BITCHENER, J. y KNOCH U. (2008). The value of written corrective feedback for migrant and international students. Language Teaching Research, 12, pp. 409-431.

BITCHENER, J. y KNOCH U. (2009a). The relative effectiveness of different types of direct written corrective feedback. System, 37, pp. 322-329. 
BITCHENER, J. y KNOCH U. (2009b). The value of a focused approach to written corrective feedback. ELT Journal, 63, pp. 204-211.

BITCHENER, J.; YOUNG, S. y CAMERON, D. (2005). The effect of different types of corrective feedback on ESL student writing. Journal of Second Language Writing, 14, pp. 191-205.

BRUNTON, A. (2009). Designing research into the effects of grammar correction in L2 writing: not so straightforward. Journal of Second Language Writing, 18, pp. 136-140.

CARVALHO, A. M. y SILVA, A. J. (2008). O papel do conhecimento metalingüístico nos padrões de transferência no desenvolvimento da interlíngua e suas implicações pedagógicas. Portuguese Language Journal, 3. Disponível em: http://www. ensinoportugues.org/archives/

CONSELHO DA EUROPA (2003). Quadro europeu comum de referência para as línguas. Porto: ASA.

CONSELHO DA EUROPA (2001). Portfólio Europeu de Línguas. Disponível em: http://elp. ecml.at/ELP/browseportfolios/tabid/2370/language/en-GB/Default.aspx

CHANDLER, J. (2003). The efficacy of various kinds of error correction for improvement of the accuracy and fluency of L2 student writing. Journal of Second Language Writing, 12, pp. 267-296.

CHANDLER, J. (2009). Response to Truscott. Journal of Second Language Writing, 18, pp. $57-58$.

ELLIS, R., SHEEN, Y. MURAKAMI, M. y TAKASHIMA, T. (2008). The effects of focused and unfocused written corrective feedback in an English as a foreign language context. System, 36, pp. 353-371.

FERRIS, D. R. (1999). The case for grammar correction in L2 writing classes, a response to Truscott. Journal of Second Language Writing, 8, pp. 1-12.

FERRIS, D. R. (2002). Treatment of error in second language student writing. Ann Arbor: University of Michigan Press.

FERRIS, D. R. (2004). The "grammar correction" debate in L2 writing: Where are we, and where do we go from here? (and what do we do in the meantime...?) Journal of Second Language Writing, 13, pp. 49-62. 
FERRIS, D. R. (2010). Second language writing research and written corrective feedback in SLA Intersections and Practical Applications. Studies in Second Language Acquisition, 32, pp. 181-201.

FERRIS, D.R. y ROBERTS, B. (2001). Error feedback in L2 writing classes: how explicit does it need to be? Journal of Second Language Writing, 10, pp. 161-184.

GRANNIER, D. M. (2012). Revisitando a proposta heterodoxa: como prestar atenção às particularidades da língua portuguesa. Estudios Portugueses y Brasileños, 12, pp. 161-176.

HAN, Z-H. (2001). Fine-tuning corrective feedback. Foreign Language Annals, 34, pp. $582-$ 599.

MACKEY, A.; GASS, S. y MCDONOUGH, K. (2000). How do learners perceive interactional feedback? Studies in Second Language Acquisition, 22, pp. 471-497.

POLIO, C. (2012). The relevance of second language acquisition theory to the written error correction debate. Journal of Second Language Writing, 21/4, pp. 375-389

QI, D. y LAPKIN, S. (2001). "Exploring the role of noticing in a three-stage second language writing task". Journal of Second Language Writing, 10, pp. 277-303

SACH, R. y POLIO, C. (2007). Learners' uses of two types of written feedback on a L2 writting revision task. Studies in Second Language Acquisition, 29, pp. 67-100.

SCHMIDT, R. (1990). The role of consciousness in second language learning. Applied Linguistics, 11, pp. 129-58.

SCHMIDT, R. (2001). Attention. En P. Robinson (ed.) Cognition in Second Language Instruction. Nueva York: CUP, pp. 3-32.

SHEEN, Y. (2007). The effect of focused written corrective feedback and language aptitude on ESL learners' acquisition of articles. TESOL Quarterly, 41, pp. 255-283.

STORCH, N. (2010). Critical Feedback on Written Corrective Feedback Research. IJES, vol. 10/2, pp. 29-46.

STORCH, N. y WIGGLESWORTH, G. (2010). Learners' processing, uptake, and retention of corrective feedback on writing. Studies in Second Language Acquisition, 32, pp. 303-334. 
TRUSCOTT, J. (1996). The case against grammar correction in L2 writing classes. Language Learning, 46, pp. 327-369.

TRUSCOTT, J. (1999). The case for "the case against grammar correction in L2 writing classes". A response to Ferris. Journal of Second Language Writing, 8, pp. 111-122.

TRUSCOTT, J. (2004). Evidence and conjecture on the effects of correction: a response to Chandler. Journal of Second Language Writing, 13, pp. 337-343.

TRUSCOTT, J. (2007). The effect of error correction on learners' ability to write accurately. Journal of Second Language Writing, 16, pp. 255-272.

TRUSCOTT, J. (2009). Arguments and appearances: a response to Chandler. Journal of Second Language Writing, 18, pp. 59-60.

TRUSCOTT, J. (2010). Some thoughts on Anthony Bruton's critique of the correction debate. System, 38, pp. 329-335

TRUSCOTT, J. y HSU, A. Y. (2008). Error correction, revision, and learning. Journal of Second Language Writing, 17, pp. 292-305.

VAN BEUNINGEN, C. (2010). Corrective Feedback in L2 Writing: Theoretical Perspectives, Empirical Insights, and Future Directions. IJES, vol. 10/2, pp. 1-27.

WILLIAMS, J. (2012). The potential role(s) of writing in second language development. Journal of Second Language Writing, 21/4, pp. 321-331 


\section{ANEXO I}

Textos obtenidos en el pretest.

\section{$\underline{\text { Caso } 1}$}

Costumo acordar por volta das nove horas, embora não tenha aulas todos os dias tão cedo. Tomo o pequeno almoço, de forma relaxada porque é das refeções que mais disfruto. Depois tomo banho, arranjo-me e vou para as aulas, os dias que tenho aulas às dez. Os dia que fico em casa, até chegar a hora de ir para a universidade, $\mathrm{fa}[\mathrm{ç} / \mathrm{z}]$ o exercícios, arrumo a casa ou fazo compras. Á tarde nunca tenho aulas por tanto depois do almoço descanso uns minutos enquanto vejo televisão e converço com a minha irmã, é a minha colega de casa. Mais tarde, faço as tarefas de aula que tenha de fazer e convino com algum amigo para ir tomar café. À noite faço o jantar e vejo alguma serie ou filme na televisão, quando não tenho um livro para ler. Não tenho uma hora fixa para ir dormir, vou dormir quando não aguentar mais que costuma ser por volta da uma da manhã.

\section{Caso 2}

Normalmente acordo todos os dias às 8 da manhã. A seguir, tomo um duche, visto a roupa, tomo o pequeno-almoço e vou para às aulas. $\mathrm{O}$ caminho atè a Universidade faço-o sempre a pé, e demoro uns 20 minutos. Quando acabo as aulas, por volta das 14 da tarde, vou para casa a almoçar. Não costumo ter aulas à tarde, mas às segundas e quartas feiras sim. Acabo às 9, volto para casa, estudo um bocado ou faço alguma tarefa, janto com a minha mulher, vemos um bocadinho a televisão e depois leio um livro na cama. À uma ou 1 e 30 limpo os dentes e vou para a cama.

\section{Caso 3}

Minha jornada começa as 8:00 horas e tomo o meu café da manhã com uma torrada e alguma bolacha de chocolate. Depois do café, tomo um douche e visto-me. As 9:00 horas saio de casa e vou para as aulas na Faculdade de Letras da Universidade de Salamanca, mais ou menos fico lá até as 14:00 horas da tarde, volto para casa, almoço com a minha família e assistimos o jornal na televisão. Á tarde faço as minhas tarefas da facultade, passo as minhas notas no computador e mais ou menos estudo aquilo que aprendi na aula da manhã. As 19:30 saio de casa e vou para a academia e faço esporto, se o tempo estiver bom, vou para um parque e dou uma caminhada. Quando volto para casa, as 21:00 horas, tomo um douche de novo e as 21:30 horas lancho alguma coisa. As 22:30 horas tento procurar um filme na televisão na 
televisão e assisto junto com os meus pais. As vezes se não houver um filme, vou para o meu quarto e leio um livro. As 23:00-23:30 mais ou menos vou para a cama e durmo porque ao dia seguinte acordo cedo para voltar na faculdade.

\section{$\underline{\text { Caso } 4}$}

Costumo acordar-me cedo por volta das oito da manhã. Tomo o pequeno almoço, costumo tomar uma meia de leite e uma torrada, e por vezes um sumo de laranja. Depois do pequeno almoço vou a caixa do banho e tomo um duxe, lavo-me os dentes e depois arrumo o quarto. Por volta das dez da manhã vou para as aulas, onde fico atè as dois. Ao finali?ar às aulas, vou para casa, fazo o almoço, como e depois costumo tomar um chà vermelho. À tarde não tenho aulas, então fazo as tarefas das aulas, e depois vou com os colegas à cafetaría, pastelaria etc... Quando volto, janto alguma coisa, vejo um bocadinho a televisão e vou para o quarto. Por volta dàs doze ja estou deitada, ouço um bocadinho a radio e dormo.

\section{$\underline{\text { Caso } 5}$}

A primeira coisa que eu faço todos os dias es passear os meus cães. Despois tomo o pequeno almoço e vou as aulas. Pela tarde estudo e alguns dias faço gimnastica o passeio com a minha bici. À noite, as vezes, janto com o meu namorado, vemos televisão o alguma filme. Este é o meu dia norma da segunda a sesta feira.

\section{$\underline{\text { Caso } 6}$}

Eu sinto o despertador as oito da manha e quando vou para cozinha os meus pais ja não ficam em casa. Fico na minha cama algum tempo mais e olho os telejournais da manha. Sou como as mulheres, estou muito tempo na sala de banho, tomo um duche e escova-me os dentes. Preparo o pequeno almoço as oito e meia, leite e bolachas. Apos o pequeno almoço começo o meu viagem pela cidade. Venho até universidade onde vou as aulas e visito as caballerizas, depois torno para casa. Lá falo com a minha mãe, tomo o almoço e outra vez vou as ruas onde sempre há coisas que fazer como trabalhar o estudar mais. Finalmente às noites fico duas horas no gimnasio e apos durmo muito bem.

\section{Caso 7}

Acordo normalmente às 7 horas da manhã. Depois vou à casa de banho para tomar um duche. Sobre às 7:30 visto-me e preparo as coisas que tengo que levar para ir as aulas. 
Às 8 horas tomo o pequeno-almoço; zumo de laranja, uma torrada e uma meia de leite.

Saio normalmente às $8: 45 \mathrm{~h}$ para estar na faculdade às 9:00 $\mathrm{hr}$. Cuando chuva apanho o autocarro, mas isso acontesse muito raramente. Tenho aulas das $9 \mathrm{hr}$ da manhã até às 2 horas. Depois de haver terminado as aulas vou a fazer a compra para logo poder preparar o almoço. De tarde faço desporto o vou para o centro da cidade para combinar com alguns amigos meus. Volto para casa sobre às 21:00hr preparo o jantar e deito-me sobre às 24:00 hr. 


\section{ANEXO II}

Comentarios en el test 2

Comentário: o que estava mal e motivos (por exemplo, regra a aplicar)

\section{Caso 1}

2.- Faltaba la letra -i entre " $\mathrm{e}$ " $\mathrm{e}$ "ç", a veces dudo con ciertos sonidos.

3.- Cambio de " $\mathrm{e}$ " por " $\mathrm{i}$ ".

4.- Falta de concordancia por no estar muy atenta.

5.- Estava mal la letra " $z$ " porque se escribe con "ç".

6.- Estaba mal la acentuación pues es la suma de a + la.

7.- Es todo junto.

8.- Estaba mal la letra "ç" pues es con una "s".

\section{$\underline{\text { Caso } 2}$}

1.- Não é preciso fazer a contração às simplemente as.

2.- Leva acento agudo.

4.- Não é necessária a preposição a.

5.- Leva um apóstrofe no meio das duas palavras.

6.- Não é necessária a preposição a.

7.- Dize-se lavar os dentes, não limpar os dentes.

\section{$\underline{\text { Caso } 3}$}

1,3,4,6,8, 10,12,13,15,16 .- Acentuação.

$2,7,9,11,18$.- Ortografia.

$$
\text { 14.- Verbo. }
$$

\section{$\underline{\text { Caso } 4}$}

1.- No necesita me.

5.- De banho.

6.- No se escribe con $\mathrm{x}$.

7.- No necesita m.

11.- Sin acento.

12,14 . - No se escribe con $z$, ocurre lo mismo.

13.- La acentuación. 


\section{Caso 5}

1, 7, 10.- Está en español.

2.- Sobra una -s-

3.- Falta el guión.

4.- Falta el acento que indica preposición más artículo.

5.- No sé que preposición exige.

6, 8.- No sé como es en portugués.

9.- Es preposición más artículo.

11.- Es masculina.

12.- Falta la -1-.

13.- Es con -x-y falta guión.

\section{$\underline{\text { Caso } 6}$}

2,14.- As horas obrigam acentuar a preposição, $=$ que em 2.

3,9.- A é nasalizada.

4, 18.- Artigo obrigatório.

5.- Precisa de acentuação.

6, 13, 16.- Precisa de hífen, hífen é obrigatório.

10.- Problemas com o léxico.

11.- Não é o tempo verbal correto.

15, 25.- Palavra aguda terminada em -s. precisa de acentuação, $=$ que 15.

17.- As palavras terminadas em -agem são femininas.

19, 21.- À dupla A.

22.- O é a disjuntiva espanhola não portuguesa.

23.- Expresa-se em singular.

24.- Falsa esdrújula.

\section{Caso 7}

1, 3, 12, 13.- Acentuação.

2, 5.- Interferença com os espanhol.

6.- Conjuntivo.

8, 9, 10.- Gramática.

11.- Falta dum "o".

Recebido: 10/02/2015

Aceito: 20/07/2015 